\title{
Adaptive Protection Architecture for the Smart Grid
}

\author{
I. Abdulhadi, F. Coffele, A. Dyśko, Member, IEEE, C. Booth and G. Burt, Member, IEEE
}

\begin{abstract}
Unique and varied power system conditions are already being experienced as a result of the deployment of novel control strategies and new generation and distribution related technologies driven by the smart grid. A particular challenge is related to ensuring the correct and reliable operation of protection schemes. Implementing smarter protection in the form of adaptive setting selection is one way of tackling some of the protection performance issues. However, introducing such new approaches especially to safety critical systems such as protection carries an element of risk. Furthermore, integrating new secondary systems into the substation is a complex and costly procedure. To this end, this paper proposes an adaptive protection architecture that facilitates the integration of such schemes into modern digital substations which are a staple of smart grids. Functional features of the architecture also offer powerful means of de-risking schemes and flexible implementation through self-contained modules that are suitable for reuse. An example adaptive distance protection scheme is presented and tested to demonstrate how the architecture can be implemented and to highlight the architecture's novel features.
\end{abstract}

Index Terms--Adaptive relaying, smart grids, scheme architecture and real-time digital simulation

\section{INTRODUCTION}

$\mathrm{S}_{\mathrm{a}, \mathrm{tan}}$ HORTFALLS in power system protection performance are being experienced at both transmission and distribution networks. These are due to a number of factors including increased penetration of distributed generation, varied operational conditions and severe wide area disturbances [13]. Maintaining acceptable protection performance is essential for a functional smart grid as these schemes ensure the reliable and safe operation of the primary system. Adaptive protection (whether using settings groups or more advanced setting calculation techniques) has been proposed as a solution to enhancing the performance of protection schemes in response to many of these factors [4]. However, a body of work tackling adaptive protection schemes from an architectural point of view and ultimately the verification and validation $(\mathrm{V} \& \mathrm{~V})$ of such schemes is virtually non-existent.

This paper proposes a unique requirements-driven adaptive protection architecture. Devising such an architecture not only defines the constituents of an effective adaptive protection

This research was supported by the UK Research Councils' Energy Programme as part of the Supergen FlexNet Consortium, grant no. $\mathrm{EP} / \mathrm{E} 04011 \mathrm{X} / 1$.

The authors are with the Institute for Energy and Environment, Electronic and Electrical Engineering Department, University of Strathclyde, Glasgow, G1 1XW, UK. (e-mail: iabdulhadi@eee.strath.ac.uk). scheme, but it is instrumental in delivering straightforward substation integration and effective scheme V\&V. This architecture and its constituent functions aim to contribute to the set of tools and approaches that form a "toolbox of proven technical solutions" for the smart grid [5], [6]. As communications system become more widespread and a cornerstone of delivering smart grid functions [7], emerging protection technologies (including adaptive protection) must effectively make use of these communications systems while maintaining reliable operation and interoperability between different vendor solutions.

The fact that adaptive protection essentially relies on modifying its behavior based on the prevailing power system conditions [8] raises a number of concerns related to the validity of adaptive behavior, the nature and amount of information required to infer the system state and the appropriate scheme performance at any given time. These issues compared to conventional protection philosophies, present a challenge in terms of devising suitable scheme implementations and $\mathrm{V} \& \mathrm{~V}$ procedures. The work in [9] highlights the importance of dedicated 'testing environments' as an effective means of tackling this issue which requires considering the impact of varied primary system operational conditions dictated by smart grid controls.

This paper first of all discusses the different life-cycle stages of a protection scheme or platform from the point of view of utilities and manufacturers respectively. This is critical to understanding the requirements necessary to develop an adaptive protection solution that meets the utilities' expectations while not leading to a costly and impractical overhaul of secondary substation infrastructure. In light of these requirements, the paper then develops the proposed architecture with its distinct functional layers. Detailed architecture functionality is also discussed. Challenges of integrating adaptive protection schemes into modern substations are identified and the role of the architecture in overcoming these is discussed.

Finally the V\&V of adaptive protection schemes, although not the focus of this paper, is an important issue that needs to be addressed. Some of the challenges associated with this are discussed and the inherent architectural features that facilitate scheme verification and validation are explained. Furthermore, an example adaptive distance protection scheme is presented to illustrate the validation process and demonstrate how the architecture can be physically implemented. 


\section{FunCTIONAL AND USER REQUIREMENTS FOR AN ADAPTIVE PROTECTION SCHEME}

As any engineering system, adaptive protection schemes go through several life-cycle stages from specification to development and from installation to decommissioning, following a similar life-cycle to that of conventional protection schemes. Different stakeholders have specific requirements that must be reflected in each of these stages. The main stakeholders that define these requirements are the users (power utilities) and the suppliers (manufacturers) of the adaptive protection scheme.

\section{A. Protection Scheme Life-Cycle Stages}

From the user's point of view, there are four main lifecycle stages:

- Scheme specification and design: this is dealt with on a case by case basis and draws heavily from the utility's protection policy documentation. At this stage detailed settings are calculated and the scheme logic is designed.

- Scheme installation and commissioning: schemes supplied by the manufacturer, which are assumed to be type approved, are subjected to factory acceptance tests that emulate the physical scheme setup in the substation. This is followed by installation in the substation where site commissioning tests are conducted to verify electrical connections and correct protection functionality.

- Scheme operation and maintenance: erroneous scheme operation during service is diagnosed to identify the causes of incorrect behavior. Some scheme settings may require revision should the primary system be altered (e.g. reinforcement).

- Scheme decommissioning and replacement: introduction of new schemes requires due consideration for the impact on existing systems. The evolving nature of both primary and secondary systems has resulted in a mix of modern and legacy protection schemes which highlights the bespoke nature of delivering such schemes. This raises concerns about sustainability of such approaches in light of new smart grid developments and adaptive protection scheme implementations.

From the manufacturer's point of view, focus is placed on the relaying platform which ends up as part of a standard bay solution in a utility's substation. In this case, there are four equally important life-cycle stages. The main linkage between these stages and those of the utility are the specified scheme requirements (Figure 1).

It is important to note that the last three stages of the protection platform life-cycle involve rigorous testing of the outcomes against the originally specified requirements. The life-cycle stages are:

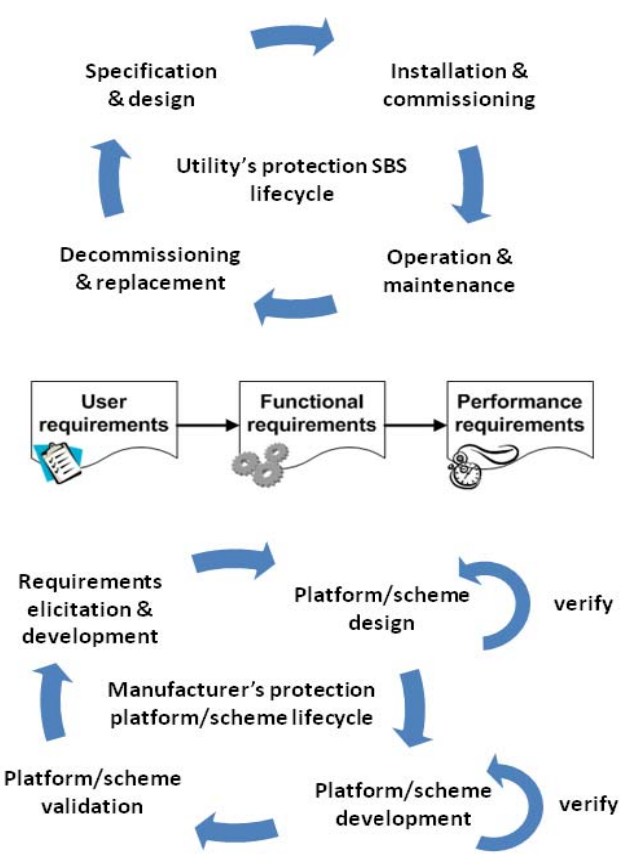

Figure 1 Protection scheme user and supplier life-cycle and requirements

- User requirements elicitation and development of functional requirements: working closely with the user ensures delivering protection platforms and schemes that are suitable. These requirements must also be developed further into performance requirements that demarcate the boundaries of the protection scheme behavior.

- Platform and scheme design: platform architectures are devised to deliver the functional requirements. Considerations are also made into mechanisms of delivering the required functionality through the platform.

- Platform and scheme development: this is a hardware and software intensive engineering process which creates and integrates the elements necessary to deliver the required functionality within the performance requirements.

- Platform and scheme validation: verification tests are conducted during the second and third stages to check that each of the elements created meet specified requirements. Once these elements are integrated as a complete platform, it is validated as a while and for being fit for purpose. A similar validation exercise is conducted to test schemes that are prepared for a customer utility.

As manufacturer's protection platforms reach maturity, the influence of the user requirements, should they change, have little impact on what the platform can inherently offer. In order to tackle this, modern relaying platforms have built-in flexibility such that new functions can be achieved through firmware upgrades or elaborate scheme logic. The architecture proposed in this paper builds upon this flexibility which allows greater flexibility to integrate within existing substation arrangements and the implementation of adaptive functions without the need for an overhaul in practices or infrastructure. 


\section{B. Adaptive Scheme Functional Requirements}

Figure 2 shows a simplified functional flow of a generic adaptive protection scheme in order to achieve a suitable setting in response to a system event. Four main functions have been identified in order to deliver this at acceptable performance levels. These are: power system event detection and qualification, post-event evaluation of protection performance and post-event setting calculation and application.

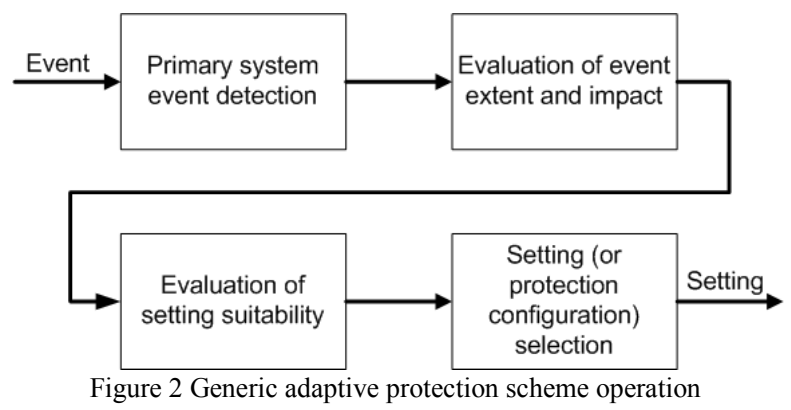

Events that have a potentially negative impact on the performance of a protection scheme must be detected through means of local or remote measurements or both. It is also necessary to qualify the extent of the event which is necessary to conduct the performance evaluation. Evaluating the performance of the protection scheme post-event is essential to ascertain the suitability of the applied settings. This function does not need to be conducted in real-time. It may be deemed sufficient to qualify the performance of the protection scheme under various operational events during the design stage which is then hardwired into the adaptive scheme. Should post-event scheme performance prove unsatisfactory after the evaluation process, a new setting is selected or calculated and then made active such that the scheme performance is brought to levels as specified by the original requirements outlined in the utility protection policy.

\section{Adaptive Scheme User Requirements}

Detailed functional requirements are highly dependent on the scheme in question and the application network. However, these serve to deliver on user requirements. The following is a brief list of the main user requirements that an adaptive protection scheme must adhere to. These should be measureable in such a way that they can be verified at different stage of scheme development:

- Utilization of existing protection functions: the essence of adaptive protection functionality is altering the configuration of existing schemes (setting or scheme logic) in order to enhance their performance. For instance, a distance scheme can be complemented with overarching adaptive functions that improve its sensitivity to in-zone faults through reach alteration.

- Primary system conditions that sanction adaptive behavior: as adaptive protection scheme do not offer a fit for all solution, it is necessary to define the events that instigate adaptive functionality and the nature of the required response. For instance, changing the topology of a distribution network may require the regarding of overcurrent protection. Information about the new topology and its onset is therefore required.

- Ease of integration with existing substation infrastructure: practical constraints posed by brown field sites and established protection practices require that adaptive schemes make most use of existing IEDs, computing and communications equipment. This manages risk of new technology as systems are decoupled and new technology introduced is limited to the adaptive functions as opposed to additional supporting infrastructure.

- Minimum performance levels met by adaptive schemes: since these schemes are meant to alleviate performance shortfalls of existing schemes, they must satisfy minimum performance levels set by utility policy. Furthermore, any single failure mode of the adaptive scheme must not cause the any further deterioration of existing protection functions.

- Standard interfaces for scheme configuration and diagnostics: adaptive protection schemes should remain faithful to the staple protection settings and scheme logic used for configuring expected scheme behavior. Furthermore signals that reflect adaptive behavior should be logged alongside disturbance records used to for postevent diagnostics. Information models adopted by the scheme should also adhere to common substation standards (e.g. IEC 61850).

All of the requirements developed above are fed in to the design process of the adaptive protection architecture proposed in following section. This aims to tackle some of the integration challenges of such new technologies and reduce their associated risks of their adoption.

\section{AdAPtive Protection ARChitecture}

One of the first and most critical steps in realizing an adaptive protection scheme is the design of a representative and functional architecture which reflects the developed requirements. This is an effective means of managing smart grid complexity through structural rigor [10]. The architecture will be referred to hereafter as the Adaptive Protection Architecture (APA). The APA serves the purpose of defining scheme elements' connectivity, their interface and the scope of their operation. Furthermore, there are functions that the architecture delivers that are thought to be important to any adaptive scheme implementation. This greatly enhances technology maturity (de-risking) through components reuse.

The proposed APA features three distinct functional layers (execution, coordination and management), as shown in Figure 3. This architecture assumes a functionally abstract form which de-emphasizes physical implementation. This allows flexibility in mapping any of the functions to suitable physical devices and platforms without sacrificing functionality which is an important feature when tackling substation integration challenges. 


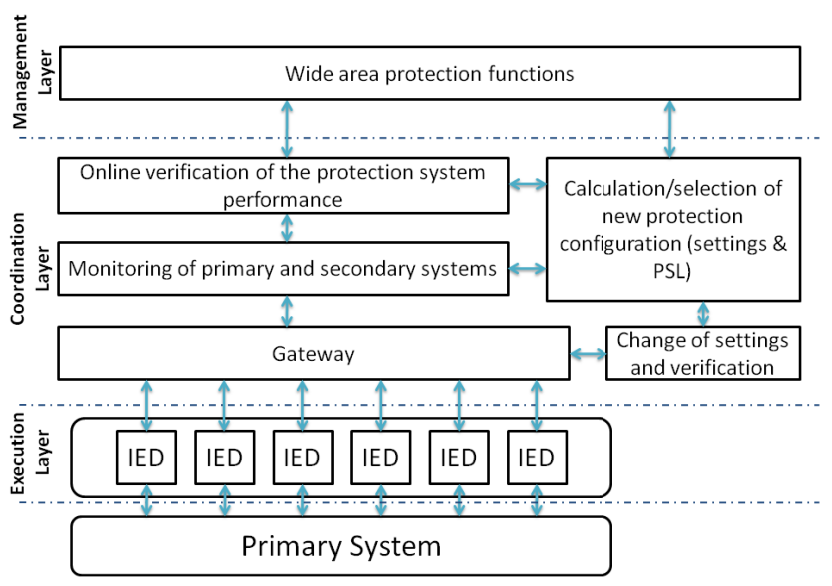

Figure 3 Adaptive protection architecture

\section{A. APA Functional Layers}

The extent of layer decoupling is determined by the local or system-wide impact of the layer's functions. Furthermore, the scope of each of the layer's functions is affected by the extent of information required to infer the state of the primary system based on which adaptive protection action is initiated.

At the execution layer, the status of the system can be usually inferred from local voltage and current measurements or by remote measurements which are defined by the scheme (e.g. digital current differential). Some local binary status information can also be used (e.g. breaker fail). Practically speaking, the execution layer encompasses modern protection IEDs. The protection functions themselves take the form of conventional protection elements such as distance protection, overcurrent protection, low frequency demand disconnection, etc. Execution layer functions usually respond to fault conditions in a matter of a few cycles depending on the protection technology and function.

The functionality scope of the coordination layer is determined simply by status information that is related to apparatus which has a direct impact on the performance of the protection scheme to be adapted. Analogue measurements aren't usually of particular use but can still be used in association with thresholds to identify unique operating conditions. Coordination layer functionality relies on this status information to evaluate the execution layer's performance under these conditions. Based on that assessment, adaptive setting selection is initiated at this level. The response of coordination layer functions is of an event triggered nature which excludes fault conditions and can take several seconds.

Finally, the management layer functions bridge the gap between the protection schemes in the field and advanced protection functions in the control room. Those are much slower in responding to events compared to the execution layer and have no direct visibility of the low level measurements. The management layer relies on measurements of a wide area nature such as synchrophasor measurements that are kept in phasor data concentrators (PDCs) to infer system state. Advanced protection functions conducted in the control room with the aid of management layer interface include diagnostic functions that evaluate the health of the adaptive (an indeed general conventional protection) scheme health. Management layer functions do not need to physically reside in the control room, but there could be regional management layer functions that are more suited to major system split points or zones that are defined by power flow or stability margins or those that are demarcated by major interconnectors. This is in contrast to suggestions found in [11], [12]. Simply handling all protection settings from the control-room as well as limiting the adaptive behaviour to system integrity protection schemes is impractical, hence the need for a detailed understanding of the scope and required performance of protection functions and different levels. And therein lies the importance functional abstraction offered by the APA.

\section{B. Detailed APA Functional Description}

Each of the APA layers constitutes a number of functions essential to adaptive protection operation. Although the application and network to be protected heavily influence the implemented functions, maturity of the technology can be achieved through the reuse of common functions even at a higher level.

Figure 4 shows the interaction and flow of information between the different functional elements of the coordination layer and those of the adjacent layers. This remains faithful to the original functional requirements for an adaptive protection scheme shown and explained in section II. B. However, it is necessary to detail a couple of the main functions performance evaluation and setting selection.

If for example the reach of a distance scheme is affected by the presence of a FACTS device (e.g. a quad booster [13]), the presence of the device and its mode of operation can be detected by the coordination layer and the suitability of the active distance reach settings can be evaluated against the minimum reach requirements stipulated by the utility's policy. This evaluation exercise can simply take the form of a reach vs. line impedance calculations. Should the minimum reach of the scheme be violated, adaptive selection of protection settings is sanctioned such that the effect of the booster is offset.

The process of setting selection can be achieved through online setting calculations, where a new setting is calculated each time the booster changes mode and in light of the network configuration. This setting is then written into the affected protection IED. Alternatively, the new setting can be chosen from a pool of pre-calculated settings groups that can be activated by IED optical inputs or through the substation local area network (LAN). This example is reported in section VI. to demonstrate the effectiveness of the APA.

As shown in Figure 4, the scope of the coordination layer encompasses several execution layers. This effectively means that coordination layer functions oversee the performance of several protection IEDs that constitute a substations protection scheme (e.g. distance schemes). On the other end of the spectrum, the management layer functions can provide additional supporting information for the coordination layer 
from a system wide-perspective or an override/enable signal which acts to prevent adaptive operation or indeed select a more appropriate setting, should this be of the primary system's interest.

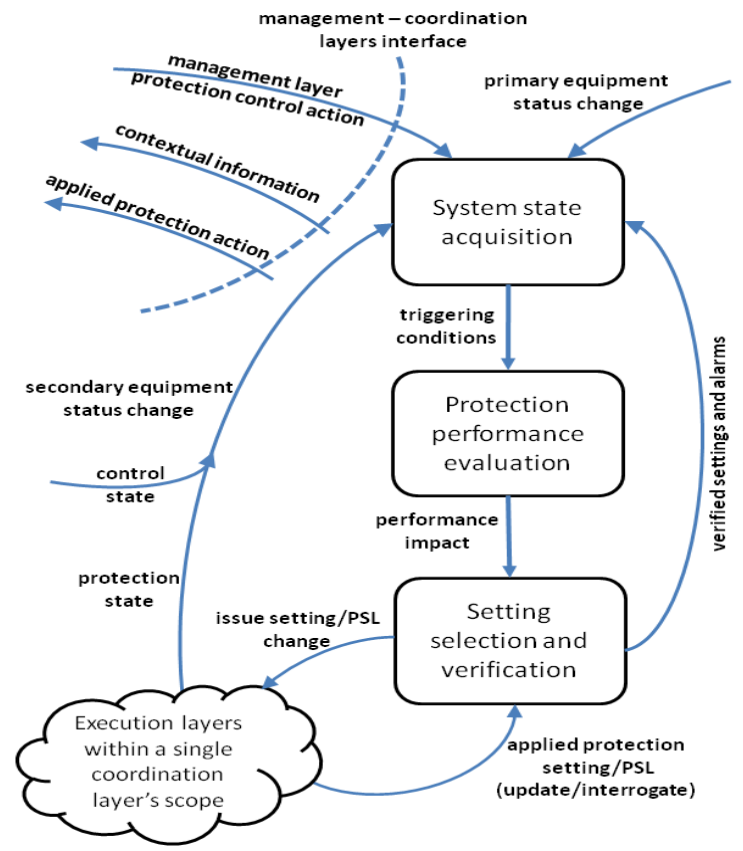

Figure 4 Detailed functional interactions at the coordination layer

There are three main functions that reside at the management layer, all of which operate with system wide protection and primary system performance in mind. These are: contextual system evaluation, protection action and protection diagnostics. Information provided by phasor measurement units (PMU), for instance, can be used by the contextual system evaluation function to determine the onset of system instability, cascade trips or major changes in power flows due to intermittent energy resources. This information is then utilized by the protection action function. For example, in response to the onset of cascade trips, low frequency demand disconnection can be targeted to operate more optimally in the network area(s) closer to the frequency deviations. This can only be achieved with wider system visibility offered at the management layer level. Another example is related to system restoration conditions where protection schemes can act against the operator [14]. Protection action functions can disable adaptive behavior or set limits for the underlying schemes such that they are desensitized to non-fault phenomena during the restoration process. Finally the diagnostics function operates in a similar manner to that of the performance evaluation function but without direct intervention. Alarms are raised for system operator action. Global logs of the adaptive behavior can also be kept for postevent analysis. Communications between the substation and regional/national control rooms plays an important role enabling these functions.

The gateway serves the purpose of providing standard communications interfaces so that different scheme elements can be integrated more easily within the substation. Plant status information and setting change requests normally pass through the gateway. Physically this can be existing substation gateways or protocol converters.

\section{Centralized vs. Distributed Functionality}

Since the APA is functionally abstract, implementing a physical scheme layout is a straightforward process of mapping functions onto physical devices. However, there are some unique physical implementation challenges from the view point of centralized and distributed layouts. Distributed functions pose a challenge since functions may need to be split over physical devices. Duplicating functions over several devices can be one way of tackling this challenge. However, there needs to be a coordinating authority through which a final adaptive setting selection decision is sanctioned.

To this end there are some functions that are suited to a certain physical layout as opposed to others. For instance, coordination functions that determine the system state and gather active setting indications from different relays are more suited to a centralized physical implementation. Conversely, active setting verification can be a distributed function, such that IEDs can verify whether they comply with the setting selection command and can raise a setting violation alarm if not.

\section{SUBSTATION INTEGRATION CONSIDERATIONS}

Considerations must be made for the communications system integration of adaptive protection schemes which constitutes several functional elements that can be from different vendors and can use different communication protocols. This challenge is being addressed by the development of substation communication gateways which can handle most of the commercial communication standards, including IEC 61850. The adaptive protection scheme can be implemented to use existing substation communication infrastructure. For example, coordination layer functions can monitor the system and change the protection settings through the communication gateway. To activate different settings groups using IEC 61850 requires writing to the SGCB control block over the LAN [15].

The proposed APA allows avoiding a major overhaul in existing substation secondary systems and infrastructure because of its flexibility, extensibility and role in de-risking novel protection functionality. These are features that are important in achieving delivering demanding performance constraints driven by smart grids [16]. Flexibility is manifested by the functional abstraction. Lack of physical constraints makes adaptive scheme implementation flexible in terms of solution offered by functions vendors and the enabling of function interoperability. Furthermore, flexibility is also a feature of smart grids which means that any adaptive protection architecture for smart grid applications must exhibit a level of flexibility. The architecture is also extensible such that additional functionality can be added without affecting the core performance of the scheme due to standalone modules. Finally, the APA play an important role of de-risking the adaptive protection functions by promoting reuse which is enabled, once again, through the functional abstraction. 


\section{SCHEME VALIDATION MECHANISMS}

The validation of protection schemes prior to their installation is of vital importance to avoid undesired malfunctioning, such as false tripping, blinding of the protection, etc. Traditional protection schemes are normally validated through a series of type tests to demonstrate that the protection scheme is capable of protecting the network under specified fault conditions.

Dynamic type tests are based on the use of transients for testing protective relays in order to simulate the dynamic behavior of the network during faults and test the protection system performance [17], [18]. Dynamic type tests have been used successfully for several years to validate traditional protection schemes, but the addition of adaptability of the protection makes the current validation techniques ineffective.

This can be explained by examining the dynamic type test process. This process involves the set up of the protection relays or the protection scheme, and is then followed by the validation process which requires the simulation of a series of transients which can be:

- Obtained from recorded or calculated waveforms: a test set can be used to playback a recorded waveform or to generate a calculated waveform that represents a transient of the network.

- Calculated in real time: an RTDS is used to simulate the network during normal and fault conditions to create a hardware in the loop (HIL) simulation.

If the protection scheme is adaptive, the protection settings are not fixed but can change as the primary system state changes. More effective validation mechanisms are therefore necessary. Knowledge of an adaptive scheme's functional blocks and their interactions, as shown in Figure 3 facilitates the formulation of a validation methodology suitable for all adaptive protection schemes that adopt the APA presented in this paper. Each of the interfaces and the functional components must adhere to the performance levels set out in the requirements. However, since the APA enables modular implementation of an adaptive scheme, replacing functions should only require the verification of these new functions.

In terms of integrated scheme validation, scenario-based HIL simulation is considered a powerful method of achieving that. Dedicated testing environments can be setup for this purpose to investigate the impact of smart grid controls on the network and in turn the protection performance [9]. And the example adaptive scheme presented in section VI. utilizes a HIL testing environment for this purpose.

\section{EXAMPle Adaptive Distance Protection System}

This section presents an example adaptive distance protection scheme which modifies the reach settings to compensate for the presence of a quadrature booster transformer (QB) in the circuit. The impact of QB on the reach of distance protection has been discussed in [13] along with a proposed adaptive scheme. This scheme has been developed and implemented in hardware while remaining true to the proposed APA.

\section{A. Adaptive Distance Scheme}

The adaptive protection scheme proposed by the authors in [13] to overcome distance protection under-reach has been implemented in a HIL configuration and is detailed later on. However, it is first important to examine the architectural features of this implementation.

The management layer functions in this case deal with plans to operate QBs nationally in a coordinated manner as alluded to in [13], [19]. This effectively uses the information available from the coordinated controller situated in the grid's control room to arm the adaptive scheme in anticipation of QB mode change. This increases the robustness of the scheme against spurious setting changes that are not matched by QB mode changes.

The main coordination layer functions perform the system state acquisition, protection performance evaluation and setting selection. The performance evaluation function uses the system impedance data along with the active setting to assess the scheme performance. Furthermore, a QB characteristic description forms the core of this function which quantifies the impact of the QB mode on distance reach. This characteristic description can be applied to different QB arrangements by simply modifying the characteristic coefficients. This reusability feature enables easier substation integration and facilitates technology maturity.

The strategy of adapting the relay's behavior is through the use of pre-verified settings groups. Two settings groups have been used, although more than two can be used if greater granularity is desired when the QB is in boosting or bucking modes. In order to facilitate the integration between the scheme and components from different manufacturers, one to one mapping of settings groups has been abandoned in favor of a reach error representation. This means that the output from the performance evaluation function can be decoupled from the physical relay's settings implementation. Setting up the scheme therefore involves an output stage mapping but at the same time allows greater flexibility in using relays from different manufacturers. The physical setup of the adaptive protection scheme in the HIL testing environment is shown in Figure 5. The coordination and management layer functions are mapped to the ABB COM600 substation gateway [20] which communicates to the RTDS over DNP3 [21], [22]. The execution layer functions reside in the Alstom P446 multi functional distance relay.

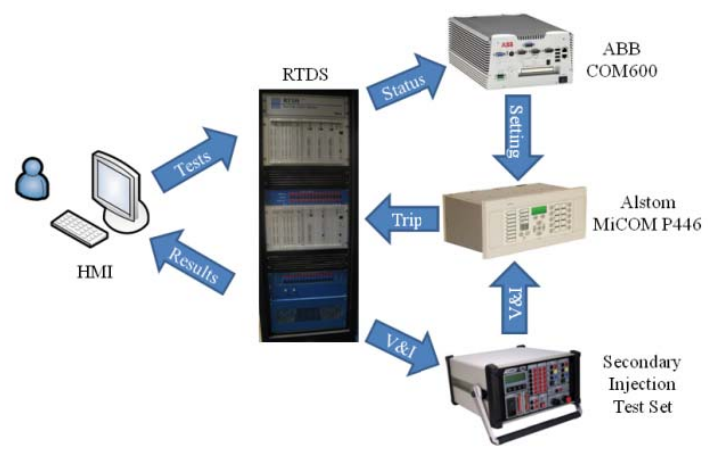

Figure 5 Adaptive distance scheme in a HIL testing configuration 


\section{B. Primary System Model}

A section of the $400 \mathrm{kV}$ UK National Grid has been modeled using RTDS as shown in Figure 6. The primary system parameters and substation designations are detailed in the appendix (section VIII. ) and [23]. A 2750MVA QB is installed on the line between HIGM and RATS and can be manually controlled during the simulation. The distance protection instrument transformers are on the line between WBUR and HIGM at WBUR substation.

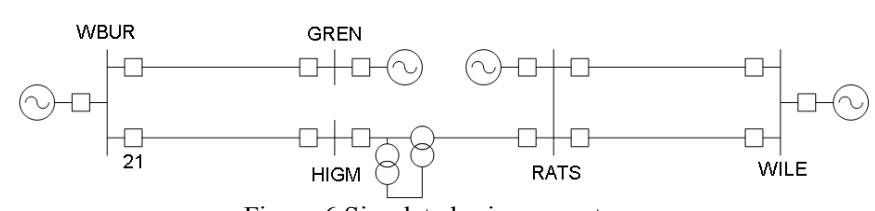

Figure 6 Simulated primary system

\section{Testing Results}

Evaluation of conventional protection distance protection performance has already been carried out in [13]. The main problem lies in zone under-reach when the QB is engaged in the circuit. Figure 7 shows an example under-reach condition for a single phase fault applied on the RATS-WILE line when the QB is in bucking mode. An exhaustive set of fault scenarios covering the full reach of the relay have been conducted to test the adaptive protection performance. The results shown here illustrate a sample of the correct operation of the adaptive scheme.

The performance evaluation function initiates a setting change from setting group 1 (SG1 signal) to setting group 2 ( $\mathrm{SG} 2$ signal) when the $\mathrm{QB}$ is engaged (QB Bypass signal) as shown in Figure 8. SG2 expands the zone reach to $20 \%$ to cover the worst case scenario. The figure also shows the correct operation of the relay when the same previously applied single phase fault is introduced to the RATS-WILE line.

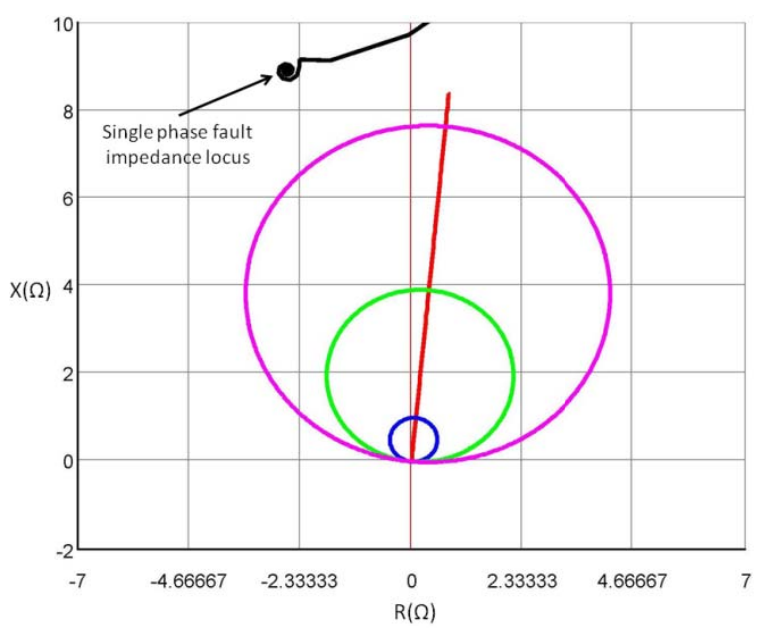

Figure 7 Non-adaptive distance protection under-reach when the QB is engaged for a single phase fault

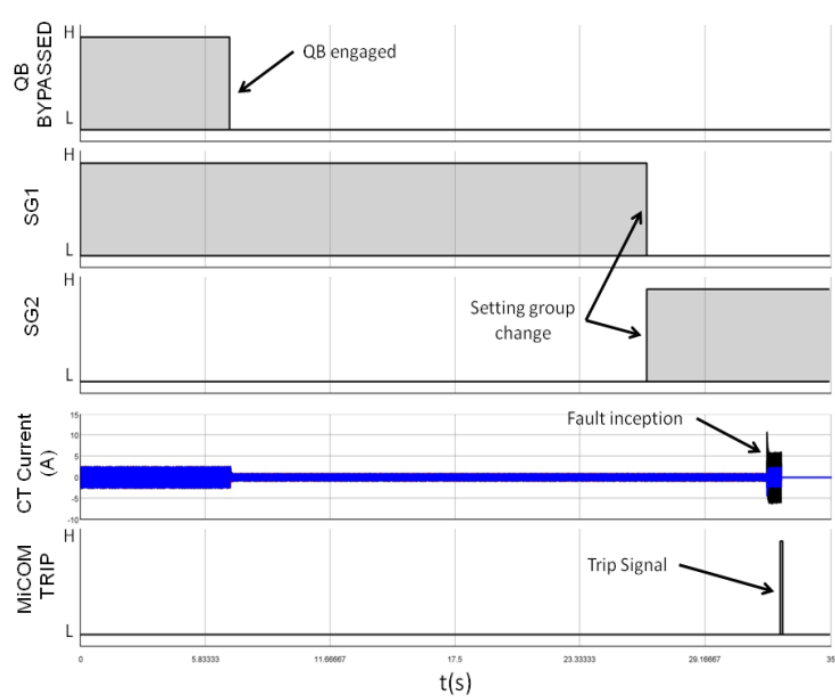

Figure 8 Correct adaptive distance protection operation when the QB is engaged and a new setting group is adapted

\section{CONCLUSION}

This paper presented a flexible adaptive protection architecture fit for smart grid applications. The architecture is requirements-driven such that it reflects utility and relay manufacturer user and functional requirements at different life-cycle stages of a scheme.

The architecture features functionally abstract adaptive and supporting functions which facilitates integration into modern digital substations as well as the de-risking of new functions by enabling their reuse.

Physical mapping of the architecture's adaptive functions has been demonstrated in a prototype adaptive distance scheme targeting transmission circuits with quadrature boosters. The correct performance of the scheme was also demonstrated in a hardware-in-the-loop based validation procedure on a transmission system whose flexible operation has been enabled through the implementation of coordinated quadrature booster control.

\section{APPENDIX - RTDS MODEL DATA AND PROTECTION SETUP}

Primary system information including line parameters and fault levels data is available in the National Grid seven year statement [23]. The source impedances were calculated using a $12 \mathrm{X} / \mathrm{R}$ ratio [24]. A 1000:1 and $400 \mathrm{kV}: 110 \mathrm{~V} \mathrm{CT}$ and CVT ratios were used respectively. Standard distance zone protection settings were used for the conventional protection (i.e. $80 \%$ zone $1,150 \%$ zone 2 , etc.). Time delays of 0 s, $200 \mathrm{~ms}$ and $600 \mathrm{~ms}$ were used for zones 1,2 and 3 respectively. Power swing blocking was enabled while other protection functions were disabled. 


\section{REFERENCES}

[1] S. K. Salman and I. M. Rida, "Investigating the impact of embedded generation on relay settings of utilities electrical feeders," Power Delivery, IEEE Transactions on, vol. 16, no. 2, pp. 246 -251, Apr. 2001.

[2] M. Khederzadeh and T. S. Sidhu, "Impact of TCSC on the protection of transmission lines," Power Delivery, IEEE Transactions on, vol. 21, no. 1, pp. 80-87, 2006.

[3] S. Horowitz, D. Novosel, V. Madani, and M. Adamiak, "System-wide Protection," Power and Energy Magazine, IEEE, vol. 6, no. 5, pp. 3442, 2008.

[4] D. Tholomier, D. Paraiso, and A. Apostolov, "Adaptive protection of transmission lines," in Power Systems Conference, 2009. PSC '09. 2009, pp. 1-14.

[5] European Commission, "European SmartGrids Technology Platform Vision and Strategy for European Electricity Networks of the Future." 2006. [Online] Available: http://www.smartgrids.eu..

[6] E. M. Davidson, V. M. Catterson, and S. D. J. McArthur, "The role of intelligent systems in delivering the Smart Grid," in 2010 IEEE Power and Energy Society General Meeting, 2010, pp. 1-6.

[7] T. Sauter and M. Lobashov, "End-to-End Communication Architecture for Smart Grids," Industrial Electronics, IEEE Transactions on, vol. 58, no. 4, pp. 1218-1228, 2011

[8] CIGRE, "Adaptive Protections and Control - Final Report," 1995.

[9] P. McLaren et al., "Testing the "smarts' in the smart T \& D grid," in Power Systems Conference and Exposition (PSCE), 2011 IEEE/PES, 2011, pp. 1-8.

[10] K. Moslehi and R. Kumar, "A Reliability Perspective of the Smart Grid," IEEE Transactions on Smart Grid, vol. 1, no. 1, pp. 57-64, Jun. 2010.

[11] Fangxing Li et al., "Smart Transmission Grid: Vision and Framework," IEEE Transactions on Smart Grid, vol. 1, no. 2, pp. 168-177, Sep. 2010.

[12] N. Markushevich, "Cross-cutting Aspects of Smart Distribution Grid Applications," in 2011 IEEE Power and Energy Society General Meeting, 2011, pp. 1-8.

[13] I. F. Abdulhadi, G. M. Burt, A. Dysko, R. Zhang, and J. Fitch, "The evaluation of distance protection performance in the presence of Quadrature Boosters in support of a coordinated control strategy," in Developments in Power System Protection (DPSP 2010). Managing the Change, 10th IET International Conference on, 2010, pp. 1-5.

[14] T. S. Sidhu et al., "Protection issues during system restoration," Power Delivery, IEEE Transactions on, vol. 20, no. 1, pp. 47-56, 2005.

[15] "Communication networks and systems for power utility automation. Basic information and communication structure. Abstract communication service interface (ACSI)," IEC Std 61850-7-2, 2010.

[16] V. M. Catterson, E. M. Davidson, and S. D. J. McArthur, "Embedded Intelligence for Electrical Network Operation and Control," IEEE Intelligent Systems, vol. 26, no. 2, pp. 38-45, Apr. 2011.

[17] ALSTOM, "Network Protection and Automation Guide - Protective Relays, Measurement and Control." 2011.

[18] "IEEE Guide for Power System Protection Testing," IEEE Std C37.2332009, pp. $1-112,2009$.

[19] M. Belivanis and K. R. W. Bell, "Coordination of phase-shifting transformers to improve transmission network utilisation," in Innovative Smart Grid Technologies Conference Europe (ISGT Europe), 2010 IEEE PES, 2010, pp. 1-6.

[20] ABB Oy, "ABB COM600 Station Automation - Product Guide," 2009. [Online]. Available: http://www.abb.com.

[21] P. Forsyth, T. Maguire, and R. Kuffel, "Real time digital simulation for control and protection system testing," in Power Electronics Specialists Conference, 2004. PESC 04. 2004 IEEE 35th Annual, 2004, vol. 1, pp. 329-335 Vol.1.

[22] RTDS Technologies, "RTDS Hardware Manual - GTNET Card.” 2009.

[23] National Grid Electricity Transmission plc, "National Electricity Transmission System (NETS) Seven Year Statement," 2011. [Online]. Available: http://www.nationalgrid.com/uk/Electricity/SYS/current/.

[24] N. Tleis, "Power Systems Modelling and Fault Analysis: Theory and Practice. Newnes," 2008.

\section{BIOGRAPHIES}

Ibrahim Abdulhadi obtained the MEng degree at the University of Strathclyde in 2007 . He is currently studying for a $\mathrm{PhD}$ in power systems. His research interests include power system protection, real-time simulation and testing and communications applications for power systems.

Federico Coffele received the B.Eng and M.Eng from the University of Padova, Italy. He is a Research Associate within the Institute for Energy and Environment at the University of Strathclyde, Glasgow, U.K and he is studying towards his $\mathrm{PhD}$ degree. His research interests include power system simulation and power system protection.

Adam Dyśko (M'06) received the M.Sc. degree from the Technical University of Łódź, Poland, in 1990, and the PhD. degree from the University of Strathclyde, Glasgow, U.K., in 1998. Currently, he is a Lecturer in the Department of Electronic and Electrical Engineering, University of Strathclyde. His main interest areas are power system modeling and simulation, power system protection, and power quality.

Campbell Booth received the B.Eng. (Hons) and $\mathrm{PhD}$. degrees from the University of Strathclyde, Glasgow, U.K. He is a Senior Lecturer within the Institute for Energy and Environment at the University. His research interests include power system protection and plant condition monitoring.

Graeme Burt (M'95) received the B.Eng. and $\mathrm{PhD}$. degrees from the University of Strathclyde, Glasgow, U.K. He is a professor within the Department of Electronic and Electrical Engineering at the University of Strathclyde, where he co-directs the Institute for Energy and Environment and he is the director of the Rolls-Royce University Technology Centre in Electrical Power Systems. 